\title{
Intersurgeon Variability in the Selection of Anterior and Posterior Commissures and Its Potential Effects on Target Localization
}

\author{
Srivatsan Pallavaram ${ }^{a}$ Hong Yu ${ }^{b}$ John Spooner ${ }^{b}$ Pierre-François D'Haese ${ }^{a}$ \\ Bobby Bodenheimer $^{\mathrm{a}}$ Peter E. Konrad ${ }^{\mathrm{b}}$ Benoit M. Dawant ${ }^{\mathrm{a}}$ \\ a Department of Electrical Engineering and Computer Science, Vanderbilt University, and \\ ${ }^{b}$ Department of Neurosurgery, Vanderbilt University Medical Center, Nashville, Tenn., USA
}

\section{Key Words \\ Intersurgeon variability · Anterior and posterior \\ commissures - Target localization - Deep brain stimulation}

\begin{abstract}
Background: This study reports the intersurgeon variability in manual selection of the anterior and posterior commissures ( $A C$ and $P C$ ). The study also investigates the effect of this variability on the localization of targets like the subthalamic nucleus, ventralis intermedius nucleus and globus pallidus internus. The additional effect of variation in the selection of the mid-plane on target localization is also evaluated. Methods: 43 neurosurgeons (38 attendings, 5 residents/ fellows) were asked to select the AC and the PC points (as routinely used for stereotactic neurosurgical planning) on two MRI scans. The corresponding mid-commissural points (MCPs) and target coordinates were calculated. Results: The collected data show that the MCP is more reliable than either the AC or the PC points. These data also show that, even for experienced neurosurgeons, variations in selecting the $A C$ and the $P C$ point result in substantial variations at the target points: $1.15 \pm 0.89 \mathrm{~mm}, 1.45 \pm 1.25 \mathrm{~mm}, 1.21 \pm 0.83$ for the subthalamic nucleus, ventralis intermedius nucleus, and globus pallidus internus, respectively, for the first MRI volume and $1.08 \pm 1.37 \mathrm{~mm}, 1.35 \pm 1.71 \mathrm{~mm}, 1.12 \pm 1.17 \mathrm{~mm}$ for the same structures for the second volume. These variations are
\end{abstract}

\section{KARGER}

Fax +41613061234

E-Mail karger@karger.ch

www.karger.com
(C) 2008 S. Karger AG, Basel www.karger.com/sfn larger when residents/fellows are included in the data set. Conclusions: The data collected in this study highlight the difficulty in establishing a common reference system that can be used to communicate target location across sites. It indicates the need for the development and evaluation of alternative normalization methods that would permit specifying targets directly in image coordinates or the development of improved imaging techniques that would permit direct targeting.

Copyright $\odot 2008$ S. Karger AG, Basel

\section{Introduction}

Modern stereotactic functional neurosurgical procedures utilize a coordinate system referenced on the anterior commissure (AC) and posterior commissure (PC) points. Based on the standard convention of the Schaltenbrand-Wahren atlas, AC and PC are defined as two points with the shortest intraventricular distance between the commissures [1]. Some neurosurgeons continue to use this definition of AC-PC based on ventriculography and true lateral skull X-rays. However, most stereotactic neurosurgery relies on MRI today, and some neurosurgeons use the center of the commissure versus the intraventricular edge of the commissure to designate the AC and PC points. Because the selection of the AC and PC points de- 


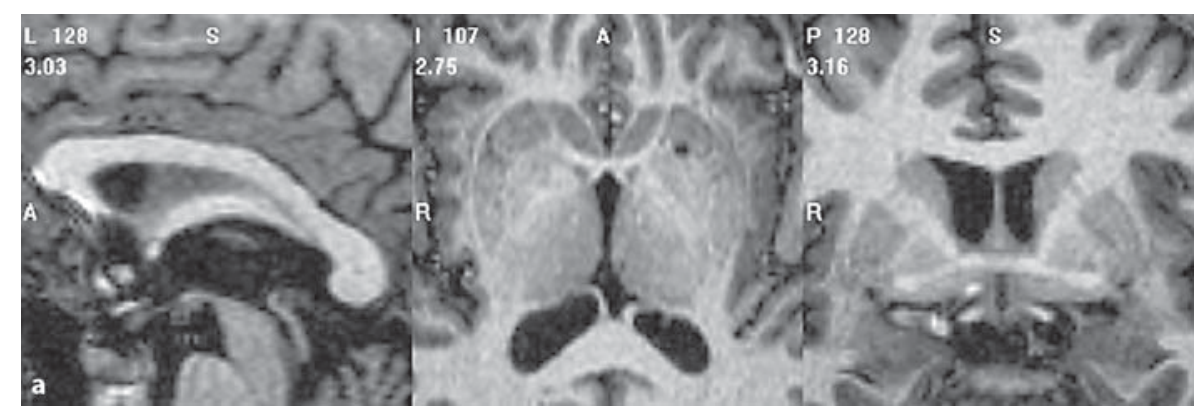

Fig. 1. a Sample display from the image viewing software. The number at the top in the upper-left corner of each view refers to the slice number and the bottom number refers to the zoom factor. $\mathrm{L}=\mathrm{Left} ; \mathrm{R}=$ right; $\mathrm{I}=$ inferior; $\mathrm{S}=$ superior; $\mathrm{A}=$ anterior; $\mathrm{P}=$ posterior. $\mathbf{b}$ Illustration of the computation of surgeon-pairwise distances between surgeon selections. b

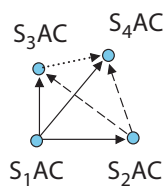

$S_{1} A C, S_{2} A C, S_{3} A C$ and $S_{4} A C$ represent the $A C$ selections by 4 surgeons $S_{1}, S_{2}, S_{3}$ and $S_{4}$.

The following surgeon-pairwise combinations can be formed with the AC selections of the four surgeons:

$\left(S_{1} A C, S_{2} A C\right),\left(S_{1} A C, S_{3} A C\right),\left(S_{1}, A C, S_{4} A C\right)$ indicated by the solid arrow $\longrightarrow$

$\left(S_{2} A C, S_{3} A C\right),\left(S_{2} A C, S_{4} A C\right)$ indicated by the dashed arrow

$\left(S_{3} A C, S_{4} A C\right)$ indicated by the dotted arrow

Distance between the two points in each pair is called a surgeon-pairwise distance. For $n$ points there are ${ }^{n} C_{2}=n \times(n-1) / 2$ such pairwise combinations and hence pairwise distances. The mean and standard deviation of these distances are computed as a measure of the intersurgeon variation in the selection of the AC. The same is done for the PC, MCP, STN, Vim and GPi. fines the reference system by which stereotactic coordinates are communicated in the literature and among surgeons, it is important to quantify errors that may occur in this reference system because of differences in visual localization of AC and PC points. Furthermore, any discussion of the localization of targets like the subthalamic nucleus (STN), ventralis intermedius nucleus (Vim) and globus pallidus internus (GPi) based on AC-PC is limited by the variability of visually selected AC and PC points. We examined the variability of manual AC-PC selections by 43 neurosurgeons who specialize in stereotactic neurosurgery as well as evaluated its impact on the localization of three popular deep brain stimulation targets.

\section{Methods}

During the American Society for Stereotactic and Functional Neurosurgery conference held in Boston, Mass., USA, in 2006, 43 neurosurgeons ( 38 attendings and 5 residents/fellows) selected $\mathrm{AC}$ and $\mathrm{PC}$ points (as they routinely do for surgical planning) on two high-resolution MRI volumes. The scans were displayed on a laptop computer with image viewing software containing simple tools for slice selection, zooming, and point selection. Both MRI volumes were acquired as sagittal $\mathrm{T}_{1}$-weighted sequences (MRI volume 1 with $1 \times 1 \times 1.2 \mathrm{~mm}$ and MRI volume 2 with
$1 \times 1 \times 1 \mathrm{~mm}$ resolution, TR $8.05 \mathrm{~ms}$, TE $3.68 \mathrm{~ms}$ ) on a 1.5-tesla Philips Medical Systems scanner. Both scans were displayed to the neurosurgeons at a resolution of $1 \times 1 \times 1 \mathrm{~mm}$ in three standard views (axial, sagittal, and coronal) simultaneously. A point selection made on one view was also displayed on the other two views to allow further refinement on any of the three views. Figure la shows a snapshot of one of the scans as shown to the neurosurgeons. Every attempt was made to allow the surgeons to select the AC and PC points without verbal cues or assistance so that bias was minimized. Surgeons were not asked to pick the mid-plane point (MP) to limit interaction time. Instead, the MP was designated by one of the neurosurgical authors (P.E.K.) and remained the same in all measurements. The $\mathrm{x}, \mathrm{y}$, and $\mathrm{z}$ coordinates (in millimeters) of $\mathrm{AC}$ and $\mathrm{PC}$ selections were recorded and the mid-commissural point (MCP) was calculated for each neurosurgeon's pair of AC and PC points. The data consisting of the coordinates $(\mathrm{x}, \mathrm{y}, \mathrm{z})$ of the manual selections made by the neurosurgeons for each MRI volume were further divided into two sets: pointset_all including selections by all 43 neurosurgeons and pointset_attendings including selections by only the 38 attending neurosurgeons.

\section{Surgeon-Pairwise Distances as a Measure of Intersurgeon}

Variability

Traditionally, the spread of a cluster of points is measured as the mean distance from the centroid of the cluster to a given point, but this does not provide a direct measure of distances between points in the cluster. In this study, which focuses on measuring 
Table 1. Literature targets for STN, Vim and GPi with respect to the AC-PC coordinate system $[2,3]$

\begin{tabular}{lcrl}
\hline \multirow{2}{*}{$\begin{array}{l}\text { Target } \\
\text { nucleus }\end{array}$} & \multicolumn{2}{l}{ Coordinates, $\mathrm{mm}$} & \\
\cline { 2 - 4 } & vertical (origin) & lateral (origin) & AP (origin) \\
\hline STN & $-4(\mathrm{MCP})$ & $12(\mathrm{MCP})$ & $-3(\mathrm{MCP})$ \\
Vim & $0(\mathrm{MCP})$ & $12.3(\mathrm{MCP})$ & $6.3(\mathrm{PC})$ \\
GPi & $-1.4(\mathrm{MCP})$ & $20.2(\mathrm{MCP})$ & $4.9(\mathrm{MCP})$ \\
\hline
\end{tabular}

intersurgeon differences, we have opted for pairwise distances. Suppose, for instance, that $\mathrm{S}_{1} \mathrm{AC}, \mathrm{S}_{2} \mathrm{AC}, \mathrm{S}_{3} \mathrm{AC}$, and $\mathrm{S}_{4} \mathrm{AC}$ are the AC selections by 4 surgeons $S_{1}, S_{2}, S_{3}$ and $S_{4}$ and that distance (a, b) is the distance between points a and $b$, then we compute distances between the 4 surgeon selections taken pairwise, i.e., distance $\left(\mathrm{S}_{1} \mathrm{AC}, \mathrm{S}_{2} \mathrm{AC}\right)$, distance $\left(\mathrm{S}_{1} \mathrm{AC}, \mathrm{S}_{3} \mathrm{AC}\right)$, distance $\left(\mathrm{S}_{1} \mathrm{AC}, \mathrm{S}_{4} \mathrm{AC}\right)$, distance $\left(\mathrm{S}_{2} \mathrm{AC}, \mathrm{S}_{3} \mathrm{AC}\right)$, distance $\left(\mathrm{S}_{2} \mathrm{AC}, \mathrm{S}_{4} \mathrm{AC}\right)$ and distance $\left(\mathrm{S}_{3} \mathrm{AC}\right.$, $\mathrm{S}_{4} \mathrm{AC}$ ) and call these surgeon-pairwise distances. Their mean, standard deviation and median (to eliminate the effect of outliers) are computed as a measure of the intersurgeon variability in selecting the AC. This is illustrated in figure $1 \mathrm{~b}$.

Measuring the Intersurgeon Variation at AC, PC and MCP and the Resultant Variation at Targets

The method described above was applied to the AC, PC and MCP coordinates for each surgeon pair to calculate the intersurgeon variability at the commissures and at the MCP. The effect of variation in the selection of $\mathrm{AC}$ and PC on target localization was analyzed using coordinates published in the literature for STN [2], GPi [2], and Vim [3]. These standard coordinates are shown in table 1 . Using the coordinates shown in table $1, \mathrm{x}, \mathrm{y}, \mathrm{z}$ coordinates for STN, GPi and Vim targets were calculated from each neurosurgeon's AC-PC selections. To generate a 3-dimensional coordinate space, one point in the mid-plane other than AC, PC and MCP was chosen by a neurosurgeon (P.E.K.) on each MRI volume. This MP remained the same in all cases, and was used in the calculation of each of the targets in $x, y, z$ coordinates. The surgeon-pairwise distances were computed from these as a measure of the intersurgeon variability at the targets. This variability is due only to the variability in the selection of the commissures.

\section{Experiment to Estimate the Effect of Mid-Plane Tilt}

To study the effect of variations in the selection of one or more MPs, which we could not study with the data set acquired at the conference, we carried out a small experiment at our institution. On the same two volumes, we asked 2 neurosurgeons (H.Y. and J.S.) to select multiple sets of 3 points on the falx that could potentially be picked by a neurosurgeon to fit the mid-plane. The AC and PC for each of the volumes were fixed. Mid-planes were then fitted through each set of points selected on the falx for each volume. Pairwise angles between all these planes (interplane angles) were then computed to measure the variability in selecting midplanes. Based on the results of this experiment the effect of midplane tilt on target localization was studied.

Variability in Manual Selection of

Anterior and Posterior Commissures

\section{Results}

\section{Variation in AC, PC and MCP Selections}

Mean and median surgeon-pairwise distances for the AC, PC and MCP selections on MRI volumes 1 and 2 and both data sets are given in table 2 . It can be noted from the results that the median variation in the selection of the $\mathrm{AC}$ is marginally larger than the median variation in selecting the PC. Mean variation for the AC can be substantially larger than that for the $\mathrm{PC}$, especially when all the data points are pooled. Mean variation for MCP is smaller than that for AC and PC for both data sets and both MRI volumes, suggesting that the MCP shows less error than the $\mathrm{AC}$ or the $\mathrm{PC}$ as a common reference point. One reason for the MCP error to be smaller than the AC and PC error is that when selecting the AC and PC points in MRI images, individual surgeons may follow either convention intraventricular edge or middle of the commissure. If this is indeed the case, our data set should show that when the $\mathrm{AC}$ point is selected anteriorly to the mean $\mathrm{AC}$ point (i.e., the middle of the AC is targeted), the PC point should be selected posteriorly to the mean PC point (i.e., the middle of the PC is targeted). We analyzed our data to determine if such a trend was apparent and found it to be true in the majority (about 65\%) of the cases. One notes, however, that the standard deviation for the MCP remains large when all the data points are pooled (pointset_all) in both MRI volumes. The maximum values that are reported in table 2 show that serious errors are possible.

\section{Variation in the Localization of STN, Vim and GPi Targets Due to Variation in AC-PC Selections}

Mean and median surgeon-pairwise distances for the STN, Vim and GPi coordinates on MRI volumes 1 and 2 and both data sets are given in table 3 . As would be expected, the variations at the targets are larger than the variation observed at the MCP, which is used as the origin of the coordinate system. The additional error is caused by the variation in the coordinate system orientation produced by variations in selecting the $\mathrm{AC}$ and the $\mathrm{PC}$ points. Results presented in table 3 show standard deviations that are relatively large (e.g. $1.71 \mathrm{~mm}$ for the Vim on MRI volume 2) for the pointset_attendings and very large when the data sets are pooled in pointset_all (e.g. $8.61 \mathrm{~mm}$ for the GPi for MRI volume 2). Histograms of surgeon-pairwise distances are useful to visualize the spread in these distances. These are shown for the AC, PC, MCP, STN, Vim and GPi in figure 2. To eliminate the effect of outliers on the histograms, we eliminated the AC and PC points that were farther away than three times the stan- 
Table 2. Mean and median of the surgeon-pairwise distances of surgeon AC-PC selections for MRI volumes 1 and 2 on both data sets

\begin{tabular}{|c|c|c|c|c|c|c|c|c|c|c|c|c|}
\hline & \multicolumn{6}{|c|}{ Surgeon-pairwise deviation, MRI volume $1, \mathrm{~mm}$} & \multicolumn{6}{|c|}{ Surgeon-pairwise deviation, MRI volume $2, \mathrm{~mm}$} \\
\hline & \multicolumn{3}{|c|}{ pointset_all } & \multicolumn{3}{|c|}{ pointset_attendings } & \multicolumn{3}{|c|}{ pointset_all } & \multicolumn{3}{|c|}{ pointset_attendings } \\
\hline & $\mathrm{AC}$ & $\mathrm{PC}$ & MCP & $\mathrm{AC}$ & $\mathrm{PC}$ & MCP & $\mathrm{AC}$ & $\mathrm{PC}$ & MCP & $\mathrm{AC}$ & PC & MCP \\
\hline Mean & 1.92 & 2.27 & 1.47 & 1.53 & 1.45 & 0.85 & 1.44 & 2.05 & 1.26 & 1.29 & 1.41 & 0.88 \\
\hline $\mathrm{SD}$ & 1.96 & 3.92 & 2.01 & 1.44 & 1.24 & 0.42 & 1.05 & 3.46 & 1.68 & 0.77 & 1.62 & 0.78 \\
\hline Median & 1.26 & 1.23 & 0.89 & 1.21 & 1.17 & 0.81 & 1.18 & 1.08 & 0.69 & 1.16 & 1.08 & 0.65 \\
\hline Max & 8.50 & 20.50 & 13.20 & 8.50 & 7.19 & 2.23 & 5.83 & 17.07 & 10.27 & 4.46 & 9.20 & 5.63 \\
\hline
\end{tabular}

Table 3. Mean and median of the surgeon-pairwise distances of target localizations for STN, Vim and GPi based on surgeon AC-PC selections for MRI volumes 1 and 2 on both the datasets

\begin{tabular}{|c|c|c|c|c|c|c|c|c|c|c|c|c|}
\hline & \multicolumn{6}{|c|}{ Surgeon pairwise deviation, MRI volume $1, \mathrm{~mm}$} & \multicolumn{6}{|c|}{ Surgeon pairwise deviation, MRI volume $2, \mathrm{~mm}$} \\
\hline & \multicolumn{3}{|c|}{ pointset_all } & \multicolumn{3}{|c|}{ pointset_attendings } & \multicolumn{3}{|c|}{ pointset_all } & \multicolumn{3}{|c|}{ pointset_attendings } \\
\hline & STN & Vim & GPi & STN & Vim & GPi & STN & Vim & GPi & STN & Vim & GPi \\
\hline Mean & 2.64 & 2.75 & 3.31 & 1.15 & 1.45 & 1.21 & 1.45 & 1.82 & 1.54 & 1.08 & 1.35 & 1.12 \\
\hline $\mathrm{SD}$ & 6.32 & 6.09 & 8.61 & 0.89 & 1.25 & 0.83 & 2.07 & 2.83 & 2.01 & 1.37 & 1.71 & 1.17 \\
\hline Median & 1.02 & 1.24 & 1.15 & 0.94 & 1.20 & 1.02 & 0.77 & 0.95 & 0.87 & 0.73 & 0.93 & 0.81 \\
\hline Max & 32.18 & 30.78 & 42.73 & 5.24 & 7.15 & 4.61 & 9.77 & 14.15 & 13.33 & 7.83 & 10.04 & 7.01 \\
\hline
\end{tabular}

dard deviation. It can be seen that the histogram for MCP is the tightest and we calculated that it has $90 \%$ of the surgeon-pairwise distances within $1.06 \mathrm{~mm}$. The AC and PC histograms have the largest spread with $90 \%$ of the distances within 1.80 and $1.70 \mathrm{~mm}$, respectively. The STN, Vim and GPi histograms are tighter than the AC and PC histograms with $90 \%$ of their surgeon-pairwise distances within $1.24,1.67$ and $1.44 \mathrm{~mm}$, respectively.

\section{Effect of Variation of Mid-Plane}

The maximum pairwise interplane angle was found to be $1.00^{\circ}$ for MRI volume 1 and $1.70^{\circ}$ for MRI volume 2 . We found that the effect of mid-plane orientation is maximum in terms of euclidean shift on the localization of GPi (error $=0.71 \mathrm{~mm}$ ) as it is farthest away from the mid-plane laterally, followed by STN (error $=0.44 \mathrm{~mm}$ ) and then Vim (error $=0.43 \mathrm{~mm}$ ) for a $2.00^{\circ}$ tilt in the mid-plane.

\section{Discussion and Conclusions}

Our data set with 43 neurosurgeons localizing AC and $\mathrm{PC}$ on the same two MRI volumes is unique. The data we have collected show that variation in the manual selec- tion of the AC and PC can have a substantial effect on target point location. It also shows that the $\mathrm{MCP}$ is a more consistent reference point than either the AC or the PC. This is likely due to canceling of differences among neurosurgeons using different conventions.

It is noteworthy that the error in designating $\mathrm{AC}$ and PC has the most effect on targeting error located more lateral from the midline. For instance, if the ideal therapeutic target for GPi was $1.4 \mathrm{~mm}$ inferior, $20.2 \mathrm{~mm}$ lateral, and $4.9 \mathrm{~mm}$ anterior to the MCP, then two different neurosurgeons (experienced attendings in this example) on average would place their target $1.44 \mathrm{~mm}$ apart based solely on the error of determining AC and PC. This would amount to the difference between two contacts on a standard deep brain stimulation electrode array $(1.5 \mathrm{~mm}$ center-to-center distance on Model 3387 electrode lead; Medronic Neurological Inc., Minn., USA).

The MRI volumes that were used in this study are of high quality, with very limited motion artifacts because the images have been acquired with the patient under anesthesia. The data we have collected, therefore, do not address the effect of quality of the images or the effect of large variability in brain anatomy (such as ventricular size). This may have a significant impact on the surgeons' 


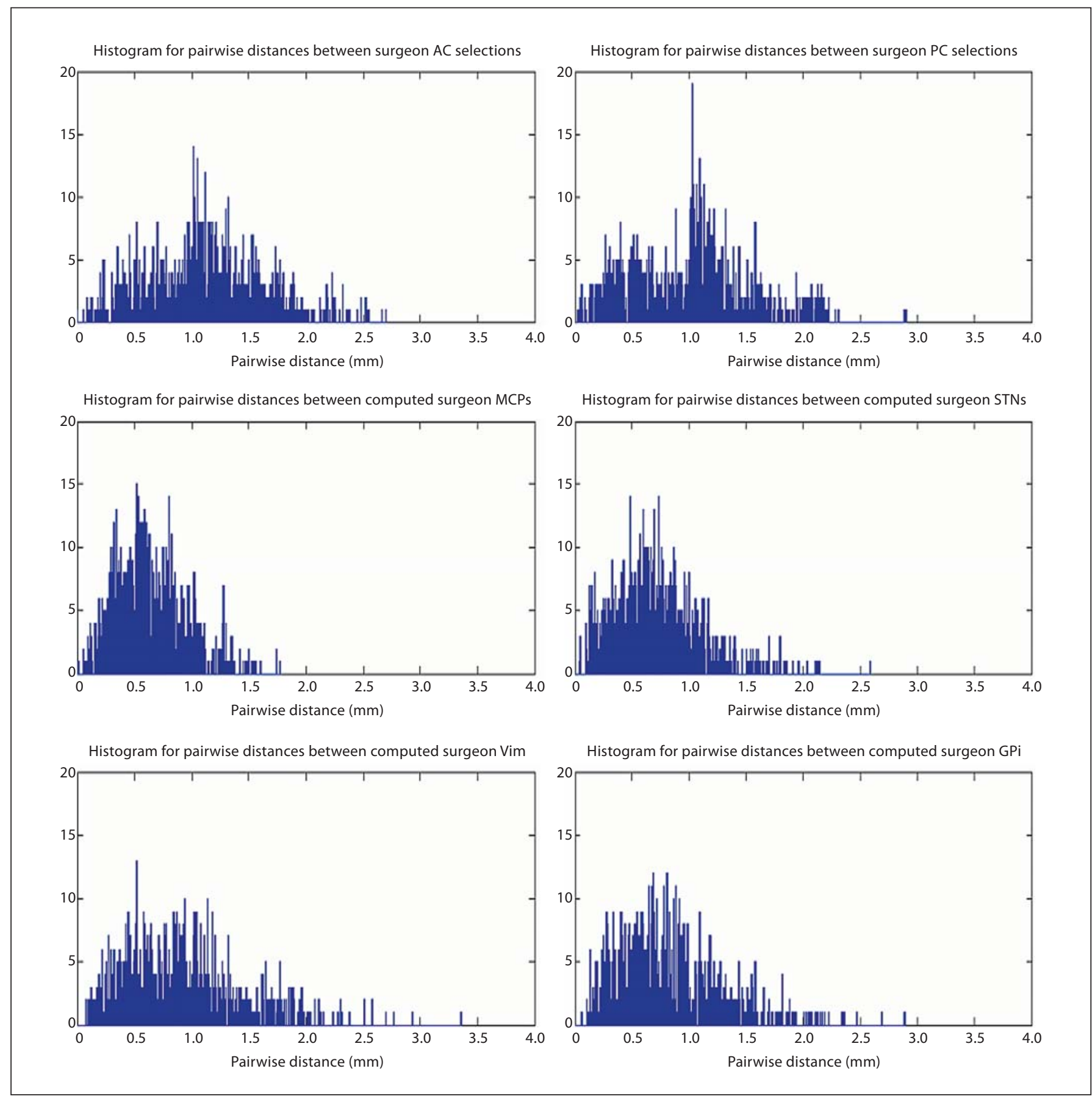

Fig. 2. Histograms for surgeon-pairwise distances $(\mathrm{mm})$ between surgeon selections of $\mathrm{AC}, \mathrm{PC}$ and the computed coordinates of MCP, STN, Vim and GPi.

ability to select AC and PC accurately. The data suggest that even with high-quality images, selection of the AC and the PC points had more variability for MRI volume 1 than for MRI volume 2. We would expect that blurring

Variability in Manual Selection of Anterior and Posterior Commissures due to motion artifacts in image volumes acquired with awake patients will worsen the results.

Our data set also strongly suggests that experience plays an important role in one's ability to select the points

Stereotact Funct Neurosurg 2008;86:113-119 
accurately. For MRI volume 1 the mean surgeon-pairwise distances for the attendings group are lower than those for the pooled group by $20 \%$ for AC and 36\% for PC. These percentages for MRI volume 2 are $10 \%$ for $\mathrm{AC}$ and $31 \%$ for PC. This translates into a mean increase in the surgeon-pairwise distance of targeting error in the pooled data (including residents/fellows) versus experienced stereotactic neurosurgeons (attendings) by 130, 90 and 174\% for the STN, Vim and GPi, respectively, for MRI volume 1. For MRI volume 2, the increases in the percentage of targeting errors between the pooled data and attendings are only 34, 35 and 37\% for the STN, Vim and GPi, respectively.

As discussed earlier, selection of the MP was held constant during the survey to reduce interaction time. Because of this, the effect of variation in selecting points on the falx on target selection could not be assessed in the subject population. However, experiments performed at our institution indicate that this could introduce an additional error of $0.71,0.44$, and $0.43 \mathrm{~mm}$ at the GPi, STN, and Vim, respectively, for a $2^{\circ}$ tilt in the mid-plane. Although this is relatively small compared to the error of AC-PC selection, we feel that this error can become more significant in patients with a curved falx. The variation results presented herein should thus be considered as a lower bound. The study we have performed highlights the difficulty in establishing a common reference system to communicate location of target points based on visual inspection of the MRI for AC-PC reference points. It suggests that a more accurate reference for stereotaxy would be to eliminate visual inspection of the AC-PC on MRI scans, and instead automate the selection based on imaging criteria. The first suggestion is to develop automatic methods that would permit the accurate and consistent localization of the AC and the PC points [4-7]. The second is to develop algorithms that permit the automatic nonrigid registration of MRI images. We have published the successful use of such algorithms to improve stereotactic localization accuracy in deep brain stimulator implant surgery [8]. This would provide normalization mechanisms that are superior to the visual inspection of MRI images and manual transcription of targets onto the Schaltenbrand-Wahren atlas. It also permits defining target points in image coordinates. An alternative approach is also being developed at other centers whereby higherresolution MRI images permit direct targeting and stereotactic localization without reliance on AC-PC coordinates. Yet, when comparing therapeutic target locations, a method of normalizing targets with respect to a common reference system (AC-PC coordinates) is highly use- ful. The source of error we have measured is only one among several sources of errors that complicates the surgical procedures. Others include the accuracy of the stereotactic frame used to place the electrode or anatomical differences between patients. It is therefore difficult to measure directly the impact of AC and PC localization errors on the overall procedure or its outcome. However, it is reasonable to believe that any source of error in the reference process could potentially lengthen the procedure by requiring more intraoperative adjustment or lead to suboptimal placements. This, in turn, could lead to less than optimal therapeutic response from the procedure.

\section{Acknowledgements}

This research has been supported, in part, by NIH grant R01EB0006136. We would also like to thank Drs. Ron Alterman, Mount Sinai School of Medicine, USA; Amed Ashraf, Kfsh University, Saudi Arabia; Hooman Azmi, University of Medicine and Dentistry of New Jersey, USA; Roy Bakay, Rush University, USA; Rick Bhasin, University of Florida, USA; Jocelyne Bloch and Claudio Pollo, Centre Hospitalier Universitaire Vaudois Lausanne, Switzerland; Joseph Christiano, Western Neurosurgery, USA; Shabbar Danish, University of Pennsylvania, USA; Milind Deogaonkar, Cleveland Clinic Foundation, USA; Mike Desaloms, Presbyterian Hospital, Dallas, USA; Jeff Elias, University of Virginia, USA; Hazem Eltahawy, Wayne State University, USA; Tiago Freitas, Hospital de Base De Brasilia, Brazil; Gary Heit, TPMG/ Kaiser Permanente, Redwood City, USA; Yannick Grenier, Jaimie Henderson and Shivanand P. Lad, Stanford University, USA; Mojgan Hodaie and Andres M. Lozano, University of Toronto, Canada; Kap Holloway, MCV, Virginia Commonwealth University, USA; Paul House, University of Utah, USA; Zelma Kiss, University of Calgary, Canada; Mark Liker, University of Southern California, USA; Carlo Marras, INNCBesta, Italy; Jules Nazzaro, Boston University Medical Center, USA; Peter Pahapill, Pennsylvania Neurosurgery and Neuroscience Institute, USA; Erik Parker, New York University, USA; Andrew Parrent, University of Western Ontario, Canada; Luiz Pereira, University of Brasilia, Brazil; Rob Plunkett, SUNY Buffalo, USA; Kim Pratt, Hackley Hospital, USA; Erich Richter, Mercer University, USA; Joshua Rosenow, Northwestern University, USA; Patrick Senatus, Columbia University, USA; Konstantin Slavin, University of Illinois, USA; Michiel Staal, University of Groningen, The Netherlands; Rodrigo Sverzickis, Pauls Stradins Clinical University Hospital, Riga, Latvia; Elizabeth Tyler-Kabara, University of Pittsburgh, USA; Osvaldo Vilela Filho, Instituto de Neurologia de Goiania, Brazil, for providing us with their selections of the anterior and posterior commissures. 


\section{References}

1 Schaltenbrand G, Wahren W: Atlas for Stereotaxy of the Human Brain with Guide to the Atlas for Stereotaxy of the Human Brain. Stuttgart, Thieme, 1977.

-2 Starr PA: Placement of deep brain stimulators into the subthalamic nucleus or globus pallidus internus: technical approach. Stereotact Funct Neurosurg 2002;79:118-145.

-3 Papavassiliou E, Rau G, Heath S, Abosch A, Barbaro NM, Larson PS, Lamborn K, Starr PA: Thalamic deep brain stimulation for essential tremor: relation of lead location to outcome. Neurosurgery 2004;54:1120-1130.
4 Pallavaram S, Yu H, Spooner J, D’Haese P-F, Koyama T, Bodenheimer B, Konrad PE, Dawant BM: Automated selection of anterior and posterior commissures based on a deformable atlas and its evaluation based on manual selections by neurosurgeons. SPIE Med Imaging Symp, San Diego, February 2007.

5 Anbazhagan P, Carass A, Bazin P-L, Prince JL: Automatic estimation of midsagittal plane and AC-PC alignment based on nonrigid registration. IEEE Int Symp on Biomed Imaging, Arlington, 2006.
6 Han Y, Park HW: Automatic brain MR image registration based on Talairach reference system. Proc Int Conf Image Processing (ICIP) 2003;1:1097-1100.

7 Vérard L, Allain P, Travère JM, Baron JC, Bloyet D: Fully automatic identification of $\mathrm{AC}$ and PC landmarks on brain MRI using scene analysis. IEEE Trans Med Imaging 1997;16:610-616

8 D'Haese P-F, Cetinkaya E, Konrad PE, Kao C, Dawant BM: Computer-aided placement of deep brain stimulators: from planning to intraoperative guidance. IEEE Trans Med Imaging 2005;24:1469-1478. 